\title{
Transatlantica
}

Revue d'études américaines. American Studies Journal

\section{Makis Solomos, De la musique au son. L'émergence du son dans la musique des $\mathrm{XX}^{e}-\mathrm{XXI}^{e}$ siècles}

Rennes, Presses Universitaires de Rennes, 2013

\section{Claude Chastagner}

\section{(2) OpenEdition}

Journals

Édition électronique

URL : https://journals.openedition.org/transatlantica/6578

DOI : 10.4000/transatlantica.6578

ISSN : $1765-2766$

Éditeur

Association française d'Etudes Américaines (AFEA)

Édition imprimée

Date de publication : 31 décembre 2013

Référence électronique

Claude Chastagner, "Makis Solomos, De la musique au son. L'émergence du son dans la musique des $x x^{e}$ xxi e siècles », Transatlantica [En ligne], 2 | 2013, mis en ligne le 25 mars 2014, consulté le 01 février 2023. URL : http://journals.openedition.org/transatlantica/6578; DOI : https://doi.org/10.4000/ transatlantica.6578

Ce document a été généré automatiquement le 1 février 2023.

\section{c) (†)}

Creative Commons - Attribution - Pas d'Utilisation Commerciale - Pas de Modification 4.0 International - CC BY-NC-ND 4.0

https://creativecommons.org/licenses/by-nc-nd/4.0/ 


\title{
Makis Solomos, De la musique au son. L'émergence du son dans la musique des $\mathrm{XX}^{e}$-XXI ${ }^{e}$ siècles
}

Rennes, Presses Universitaires de Rennes, 2013

\author{
Claude Chastagner
}

\section{RÉFÉRENCE}

SOLOMOS, Makis, De la musique au son. L'émergence du son dans la musique des XXe-XXI siècles, Rennes, Presses Universitaires de Rennes, 2013, 545 pages, ISBN 978-2-7535-2638-9, 24 euros.

1 L'ouvrage de Makis Solomos, professeur de musicologie à Paris 8 et le spécialiste de Xenakis en France, pourrait impressionner, voire alarmer le lecteur. Par son volume (plus de 540 pages denses), l'ambition de son propos (une analyse inédite de l'évolution des musiques européennes et étatsuniennes, pour l'essentiel, depuis l'aube du $\mathrm{xx}^{\mathrm{e}}$ siècle jusqu'à nos jours), son approche à la fois musicologique, philosophique et sociologique, son éclectisme et son érudition. D'autres pourraient en outre lui reprocher de s'intéresser à ce qu'ils appellent «une musique de bruits, c'est-à-dire une non musique " (92). Ce serait sans compter sur les talents de pédagogue et la finesse stylistique de Solomos, qui combine fresque historique exhaustive et analyses exigeantes en une somme lumineuse, aérienne et élégante.

2 La thèse de Makis Solomos est que la musique, savante comme populaire, s'est progressivement éloignée au cours du $\mathrm{xx}^{\mathrm{e}}$ siècle de ses constituants originels (hauteur et harmonie), remis en question par l'émergence du timbre, de la dissonance et du bruit et que ces nouveaux paramètres entraînent de nouvelles modalités de composition et d'écoute dans lesquelles le concept de «son » occupe une place centrale. L'essentiel de la démonstration de Solomos vise donc à faire comprendre ce qu'il entend par «son » et quelles sont les manifestations et les conséquences de ce changement de paradigme. 
3 Après avoir retracé l'évolution du concept de timbre, prédominant jusqu'à la fin du $\mathrm{xx}^{\mathrm{e}}$ siècle, Solomos analyse comment la prise en compte du bruit (les sons non périodiques, à hauteur non déterminée) s'est imposée, via la musique concrète et le bruitisme, bousculant les attentes et les habitudes d'écoute traditionnelles, fondées sur la norme harmonique et mélodique. Si ces normes subsistent, la priorité est dorénavant accordée au travail sur la substance sonore, en relation avec l'apparition de nouveaux instruments, du studio d'enregistrement au synthétiseur en passant par la bande magnétique. Ce changement de priorité conduit Solomos à examiner l'émergence d'une nouvelle modalité d'écoute, qu'il qualifie d'immersion sonore, déclinée en immersion océanique, immersion dionysiaque, etc. Solomos nous montre comment une telle réflexion sur le son et l'écoute amène de nombreux artistes à intégrer des préoccupations spirituelles à leur démarche. Il explore ensuite la façon dont les artistes contemporains utilisent le son non pas comme un matériau "prêt à l'emploi » dont ils se serviraient pour composer, mais comment leur musique constitue un processus d'élaboration et de construction du son, substituant «la composition $d u$ son à la composition avec des sons » (491). De fait, écrit Solomos, «l'histoire de la modernité pourrait s'écrire comme une succession de plus en plus rapprochée de nouveaux matériaux " (280), qu'il s'agisse de synthèse granulaire, d'infra-chromatisme, de paramétrisation intégrale, de sons de synthèse, etc., autant de termes qu'avec des exemples précis Solomos rend très concrets et compréhensibles pour le profane. L'idée de construction et de rapport entre textures, surfaces et masses est reprise dans le chapitre final via une réflexion sur la notion d'espace (comme lieu physique de l'écoute - quadriphonie, surround - ou espace mental de composition, jusqu'aux soundscapes de R. Murray Schafer et aux field recordings de Steven Feld). Les dernières pages évoquent les plus récents développements qui intègrent à la composition des principes architecturaux, témoins du processus de "géométrisation progressive de la musique " (437). À ce titre, les États-Unis constituent, précise Solomos, « une terre fertile pour les installations sonores » (468).

4 La place centrale qu'occupe la musique étatsunienne dans l'ouvrage de Makis Solomos justifie pleinement qu'il soit mentionné dans les pages de Transatlantica. Solomos réussit la gageure de croiser différentes formes musicales, savantes comme populaires, pour la plupart développées aux États-Unis, dans un refus d'autant plus salutaire des cloisonnements, hiérarchies et catégorisations que son récit de l'émergence du son souligne à quel point les différents courants musicaux se sont mutuellement influencés, et qu'il rappelle que les musiciens les plus grands ont pris la liberté de l'éclectisme et de l'ouverture. Solomos insiste longuement, avec raison, sur le rôle fondateur joué par Varèse, qui « rejette le clivage son musical/bruit et postule que tout son, tout bruit peut être utilisé à condition qu'il soit “ organisé" " (115), Varèse dont, faut-il le rappeler, l'un des admirateurs les plus fidèles fut Frank Zappa. Solomos insiste également sur le recentrement sur l'écoute opéré par Cage (sa célèbre formule « Happy New Ears »!) et sur les expériences sonores effectuées par La Monte Young, Alvin Lucier ou Morton Feldman. Mais auparavant, dans une démarche moins habituelle, Solomos sera remonté aux sources de la musique moderne américaine, pour décrire et analyser l'introduction au début $\mathrm{du} \mathrm{xx}^{\mathrm{e}}$ siècle de la dissonance par les «ultramodernes », ce qui leur valut le surnom de «bad boys » (104). Solomos met alors en avant le rôle politique qu'à cette époque déjà la dissonance a pu prendre, via des compositeurs comme Henry Cowell (l'inventeur de la notion de cluster), Ruth Crawford, Charles Seeger et surtout Charles Ives. 
5 Solomos développe ensuite longuement le travail de Steve Reich (dont il explore les rapports avec celui de différents artistes plasticiens), de Philip Glass, les expériences électroniques de Louis et Bebe Baron et de Milton Babbitt, et les liens qui se tissent alors, via le minimalisme, la répétition et l'usage inventif de l'électricité entre ces musiques souvent encore qualifiées de savante ou d'avant-garde, et les formes les plus innovantes de musique populaire, du rock au free-jazz, jusqu'à la new age et à l'ambient des années quatre-vingt-dix et les expériences bruitistes, voire "industrielles» de Sonic Youth ou de Glenn Branca, et les critiques radicales du capitalisme que ces expériences cherchent à produire.

6 On l'aura compris, De la musique au son n'est donc pas un ouvrage technique et aride destiné aux seuls musicologues avertis, mais l'œuvre d'un chercheur protéiforme, humaniste et érudit, qui joue autant avec les mots et leur étymologie qu'avec les sons, qui prend le temps de ralentir son récit pour consacrer de longs développements à une œuvre spécifique ou à un artiste, qui sait varier et enrichir son argumentation de nombreux extraits de sources primaires passionnantes, soigneusement commentées, et d'une abondante iconographie, en particulier de très éclairantes partitions d'œuvres contemporaines. Au point que bien souvent l'analyse musicale se transforme en une véritable métaphysique du son doublée d'une réflexion sur la fonction sociale, critique et contestataire, de ces musiques.

INDEX

Thèmes : Recensions

\section{AUTEURS}

\section{CLAUDE CHASTAGNER}

Université Paul-Valéry (Montpellier) 